OPEN

SUBJECT AREAS:

MECHANICAL

ENGINEERING

FLUID DYNAMICS

Received

31 July 2014

Accepted

21 November 2014

Published

12 December 2014

Correspondence and requests for materials should be addressed to

S.K.M. (sushanta. mitra@lassonde.yorku.

\section{Under-water superoleophobicity of fish scales}

\author{
Prashant R. Waghmare', Naga Siva Kumar Gunda² \& Sushanta K. Mitra²
}

'University of Alberta, Edmonton, Alberta, Canada, ${ }^{2}$ Micro \& Nano-scale Transport Laboratory, Department of Mechanical
Engineering, Lassonde School of Engineering, York University, Toronto, Canada.

Recent surge in the development of superhydrophobic/superoleophobic surfaces has been motivated by surfaces like fish scales that have hierarchical structures, which are believed to promote water or oil repellency. In this work, we show that the under-water oil repellency of fish scales is entirely due to the mucus layer formation as part of its defense mechanism, which produces unprecedented contact angle close to $180^{\circ}$. We have identified the distinct chemical signatures that are responsible for such large contact angle, thereby making fish scale behave highly superoleophobic inside the water medium. In absence of the mucus layer, it is found that the contact angle decreases quite dramatically to around $150^{\circ}$, making it less oleophobic, the degree of such oleophobicity can then be contributed to its inherent hierarchical structures. Hence, through this systematic study, for the first time we have conclusively shown the role of the fish's mucus layer to generate superoleophobicity and negate the common notion that hierarchical structure is the only reason for such intrinsic behavior of the fish scales.

- ish scales are excellent examples of under-water superoleophobic or oil-repellent surfaces. In fact such - repelling action of the fish scales extends to different oils, and is considered to be one of the primary reasons responsible for the survival of the fishes in polluted water ${ }^{1}$. Beyond this remarkable biophysical consequence, fish scales, like other natural liquid-repellent surfaces (such as lotus or rice leaves or butterfly wings) ${ }^{2-5}$, have inspired development of artificial surfaces that mimic the fish scale wetting behavior by reproducing the corresponding micro-nanoscale structural features present on the fish scales. Successful realization of such attempts is naturally based on the exact understanding of the factors responsible for the typical wetting behavior of the fish scales. Like most other extreme liquid-repellent surfaces ${ }^{6,7}$, the wetting behavior of the fish scales, too, results from a combination of its intrinsic material and hierarchical micro/nanostructures on its surface. In addition, and this is where the fish scales are different from other natural examples, it is believed that the slippery mucus layer present on the fish scales ${ }^{1,2,8,9}$ substantially aids in ensuring the under-water superoleophobic behavior of the fish scales. Remarkably, this entire hypothesis on the combined consequence of the mucus and the fish scale architecture in dictating the fish scale wettability has never been truly verified by testing the wettability of the fish scales with and without the mucus layer. Since fish always secretes mucus as a defense mechanism ${ }^{1}$ when it comes in contact with any foreign agent, which can be the polluting oil - a conclusive understanding about the role of mucus, in dictating fish scale wettability, is central to complete the picture of mucus as a key defense mechanism of the fishes.

Liu et al. ${ }^{5}$ measured static contact angle of fish scales both in air (showing complete wetting in air) and in water $\left(156.4 \pm 3.0^{\circ}\right.$ : degree of oleophobicity) and found that the wetting properties of these scales changed from completely oleophilic in air to superoleophobic under-water due to presence of hierarchical structures. However, their study does not pin-point the role of mucus layer towards this superoleophobic behavior. Bixler and Bhushan ${ }^{3,10}$ studied the wettability of two different species - fish scales (Oncorthynchus mykiss) and shark skins (Insurus oxyrinchus) with respect to water drop in air medium in presence and absence of mucus layer for each case. They found that for their fish scales, the static contact angle for water in air is less than $10^{\circ}$, which changes to $58^{\circ}$ on removal of the mucus layer - thereby still having the fish scales hydrophilic for water in air medium. The limited studies, reported so far, have a huge challenge in determining accurate static contact angle, particularly in situations under-water, where the pendant drop technique is often used with the needle being attached to the drop when brought in close contact to the target substrate for contact angle measurements. Coupled with this challenge is the realization of a completely flat substrate requirement for contact angle measurement, which is exceedingly difficult to achieve for bio- organisms with complex surface structures like those seen on the fish scales ${ }^{3,4,10,11}$. 
Fish, in general, brings added complexity towards understanding the hydrophilic/oleophilic characteristics, compared to other substrates commonly found in nature like lotus leaves, butterfly wings ${ }^{3-6}$, etc. because of its inherent ability to secrete slippery mucus layer as form of a defense mechanism towards any externally induced stresses, like touch, environmental pollutants, toxins in water, etc. The mucus is secreted from the cells located at the outer skin (epidermis) covering the fish scales. It is also known and well-studied that the mucus helps in reducing the drag on the fish and thereby helping fish to swim in water with less friction ${ }^{3,9,12}$.

However, as mentioned earlier, little attention has been focused on understanding the role of such mucus layer towards the wetting characteristics of the fish scales. In this work, through the underwater wettability measurement of the fish scales of Oreochromis niloticus (commonly known as Tilapia fish), with and without the mucus, we analyze the relative importance of the mucus and the fish scale architecture in dictating the under-water superoleophobicity. Here, for the first time, we are able to accurately determine the static contact angle of oil droplets on fish scale under-water using a needlefree drop deposition technique. Our results establish that presence of mucus enhances the under-water superoleophobicity. Through careful experiments conducted by removing the mucus layer from the fish scale, it emphasized that instead of the hierarchical micro/nanostructures present in the fish scales, the mucus layer plays a dominant role in changing the wetting characteristic of fish scales towards oil.

\section{Results and Discussion}

Figure 1 provides the contact angle measurements for the fish scales with and without mucus layer. It is found that in air medium, the contact angle with oil drop changes from $30.6 \pm 7.7^{\circ}$ in case of scales with mucus to $24.1 \pm 0.7^{\circ}$ for scales without mucus. However, in air medium with water drop, the contact angle is found to be $34.1 \pm 2.7^{\circ}$ with mucus, which changes to $85.7 \pm 4.7^{\circ}$ for scales without mucus (please refer to Table S1 in Supplementary Information for the magnitude of contact angles for different cases). Such increase in contact angle for water in air medium has also been reported for Oncorthynchus mykiss fish ${ }^{3,10}$. In case of water medium, with oil drop, it is found that the fish scale with mucus layer behaves as a superoleophobic surface with unprecedented contact angle $\approx 180^{\circ}$ (measured value is $178.4^{\circ} \pm 1.3$ ). Such superoleophobicity has not been determined accurately in earlier studies ${ }^{4,5,11}$ for fish scales. Through our careful mucus removal process, it is found that the same fish scales without mucus shows a reduced superoleophobic behavior, with a measured contact angle of $148.9 \pm 5.5^{\circ}$. The super-

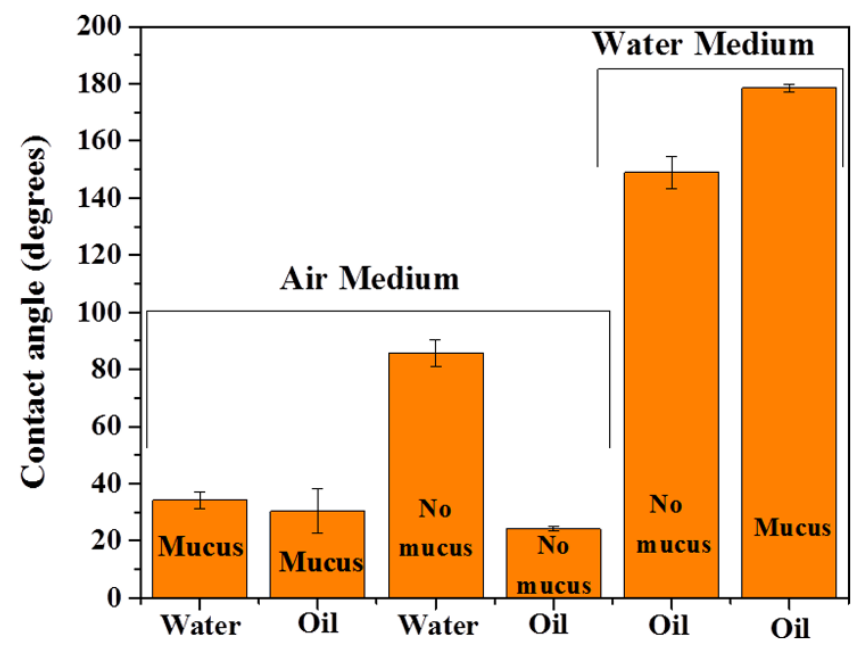

Figure 1 | Contact angle measurement on fish scales. Static contact angle of oil and water drops on fish scales with and without mucus layer for both in air and water media. oleophobicity of fish scales without mucus layer can be considered as the contribution purely due to the geometrical asperities. However, our study clearly demonstrates that such hierarchical structures cannot alone contribute to unprecedented superoleophobic behavior of fish scales under-water. The inherent mucus layer, which is produced due to external stress like a pollutant oil drop in marine ecosystem, is responsible for this added superoleophobicity of fish scales underwater. The role of surface roughness on the wetting nature of fish scales can theoretically be presented using the Cassie-Baxter and Wenzel theories where the prior information regarding roughness parameters (height, pitch, width, etc. of the individual micro feature) and equilibrium contact angle on a flat smooth surface of the same material are needed. Hence, in the case of fish scales the knowledge of the geometrical micro features of fish scale and equilibrium contact angle on the smooth fish scale is an utmost requirement. The microscopic images (SEM as presented in this study) can provide geometrical features but on the other hand obtaining perfectly flat and smooth fish scale for equilibrium contact angle measurement is impractical. Hence, in most of the cases the fish scales are mimicked on the glass, silicon or the PDMS, using cumbersome micro-nano fabrication techniques, and the role of surface roughness is studied, using Cassie-Baxter and Wenzel theories ${ }^{3,6,11}$.

Further the role of surrounding medium and wettability of substrate can be understood using mathematical argument as presents here. Let us consider a substrate in two different media i.e., in air and in water. Let us denote the interfacial surface tensions and contact angles for different combinations as $\gamma_{\mathrm{ij}}, \gamma_{\mathrm{ik}}$ and $\theta_{\mathrm{ij}}$, respectively, where $i$ and $j$ represent different phases i.e., air (a), water (w) or oil (o), and $\mathrm{k}$ represents the solid substrate.

Based on Young's equation, one can write ${ }^{13}$ :

$$
\gamma_{\mathrm{ij}} \cos \theta_{i j}^{k}=\gamma_{\mathrm{ik}}-\gamma_{\mathrm{jk}}
$$

Therefore, one can write,

$$
\gamma_{\text {ow }} \cos \theta_{\text {ow }}=\gamma_{\text {oa }} \cos \theta_{\text {oa }}-\gamma_{\text {wa }} \cos \theta_{\text {wa }}
$$

For two different substrates i.e., with and without mucus, one can obtain two different equations using Eq. (2). Further, the difference in the wettability of these two substrates can be presented as

$$
\begin{aligned}
& \cos \theta_{\mathrm{ow}}^{\mathrm{m}}-\cos \theta_{\mathrm{ow}}^{\mathrm{nm}}=\frac{\gamma_{\mathrm{oa}}}{\gamma_{\mathrm{ow}}}\left(\cos \theta_{\mathrm{oa}}^{\mathrm{m}}-\cos \theta_{\mathrm{oa}}^{\mathrm{nm}}\right) \\
& -\frac{\gamma_{\mathrm{wa}}}{\gamma_{\mathrm{ow}}}\left(\cos \theta_{\mathrm{wa}}^{\mathrm{m}}-\cos \theta_{\mathrm{wa}}^{\mathrm{nm}}\right)
\end{aligned}
$$

Here, superscript ' $n$ ' and 'nm' denote two substrates with mucus and without mucus, respectively. It is to be noted that change in the wettability due to presence and absence of mucus i.e., $\left(\cos \theta_{\mathrm{ow}}^{\mathrm{m}}-\cos \theta_{\mathrm{ow}}^{\mathrm{nm}}\right)$ is a not only a function of contact angles in air and water media $\left(\theta_{\mathrm{wa}}^{\mathrm{m}}, \theta_{\mathrm{wa}}^{\mathrm{nm}}, \theta_{\mathrm{oa}}^{\mathrm{m}}\right.$ and $\left.\theta_{\mathrm{oa}}^{\mathrm{nm}}\right)$ but it also depends on magnitudes of different interfacial tension $\left(\gamma_{\text {wa }}, \gamma_{\text {ow }}\right.$ and $\left.\gamma_{\text {oa }}\right)$. Therefore, it can be inferred that apart from the equilibrium contact angles on such surfaces, the interfacial tension also dictates the decrease in the oleophobicity.

To further delineate the effect of mucus layer on the wetting characteristics of the fish scales, we have scraped the mucus layer from the whole fish using the backside of the sterile surgical knife and collected the mucus sample on a glass side. The mucus sample was sandwiched between the two glass slides for uniform spreading of the sample and later taken apart and dipped into the glass couvette filled with DI water to measure the contact angle of oil drop on mucus layer alone. It is found that mucus layer on glass surface produces a similar unprecedented contact angle $\approx 180^{\circ}$, which further collaborates our earlier findings that mucus layer is solely responsible for such unprecedented superoleophobic behavior of fish scales (refer Supplementary Video S1). However, the mucus layer on a glass substrate looks wavy and rough as these are soft gel like material which 


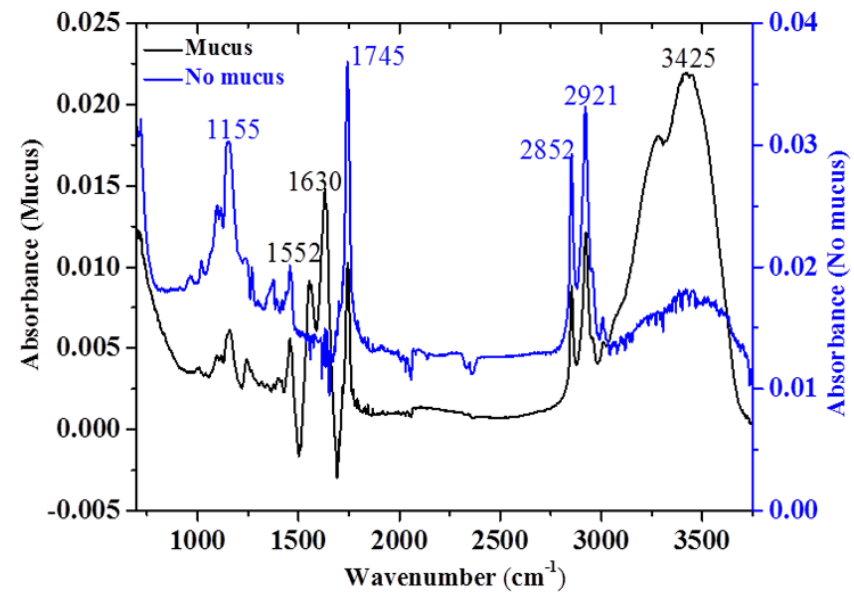

Figure $2 \mid$ FTIR data for fish scales with and without mucus along with key chemical signatures.

has confirmed to its own minimum energy state, when deposited on a flat glass substrate. The key here is to emphasise that such conformity of the mucus layer on a glass surface has no bearing on the underlying roughness of the glass substrate, which in this case is almost flat. Therefore, through such exercise (as demonstrated in Video S1), we are able to demonstrate the role of chemical signature, which dictates such oil repellency behavior.

An Attenuated Total Reflection Fourier Transform Infrared (ATR-FTIR) spectroscope was used to obtain the chemical signature of the fish scales, with and without mucus.

The chemical signature of mucus layer obtained from FTIR data, which is presented in Fig. 2, shows the presence of functional groups in the range from $1600 \mathrm{~cm}^{-1}$ to $3400 \mathrm{~cm}^{-1}$, which are hydroxyl $(-\mathrm{OH})$, amine $(-\mathrm{NH} 2)$ and carbonyl $(\mathrm{CO})^{14}$. Also, the mucus layer exhibits three distinct peaks that correspond to molecules, representing alcohols and phenols, which disappears for the mucus free sample. These functional groups along with several other components of fish mucus may lead to the formation of an oil repelling layer between the fish scale and the polluting oil through its interaction with the surrounding water medium. Due to this, the foreign oil drop virtually does not feel the presence of the underlying micro/nano-structured surface and probably creates an unfavorable spreading parameter ${ }^{13}$, which thereby produces such unprecedented superoleophobicity.

\section{Materials}

Experiments are conducted on fish scales extracted from a Tilapia fish obtained from local grocery store in Edmonton, Canada. Small portions of the fish, as shown in Fig. 3 , are sectioned out using sterile surgical knife and the underlying fish body below the dermis has been carefully removed to obtain almost flat substrates for contact angle measurement. These sections were further transferred on microscope glass slides to provide rigid support to the fish scales. In case, where mucus layer from the fish scales need to be removed, they were done prior to transferring the sections on the glass slide. Chemical reagents like Ethylenediaminetetraacetic acid (EDTA), Dithiothreiotol (DTT), phosphate buffer solution (PBS), Dipco's modified eagle medium (DMEM), Penicillin streptomycin, Gibco, Glucose are obtained from Fisher Scientific (Canada) Silicon oil (Cargille Inc., USA) and de-ionized (DI) water (obtained from PURELAB Ultra, ELGA) are used as measurement fluids for contact angle measurement using Drop Shape Analyzer DSA 100 (Krüss, Germany).

\section{Methods}

All experimental protocols were approved by Risk Management Services, University of Alberta and methods were carried out in accordance with Biosafety Officer, Environment, Health and Safety Risk Management Services, University of Alberta.

Accurate Determination of Under-water Contact Angle. One of the key challenges in measuring the static contact angle for substrates like fish scale under-water is to have a mechanism in place where the needle producing the desired oil droplet (for pendant drop technique ${ }^{15}$ ) does not remain attached to the drop once it is brought in close contact to the characterizing substrate, i.e., fish scales. When the needle is attached to the drop, it creates additional force on the drop-substrate combination, which further spreads the drop on the substrate, thereby providing erroneous contact angle values ${ }^{3,5}$. Our recent under-water needle-free drop deposition technique ${ }^{15}$ overcomes this limitation by bringing in an additional oil-water interface (as shown in Fig. 4(b) with canola oil used at the interface between water and air) and hence this has been used for accurate estimation of the static contact angle of oil drop on fish scales kept under water, for both with and without mucus, as shown in Fig. 4 (a). This accurate measurement of contact angle is more critical in this case as fish scale with or without mucus is essentially a soft deformable substrate (soft matter) and hence any force exerted by the traditional needle-drop assembly on the characterizing substrate during the contact angle measurement (as done in conventional pendant drop method) will generate additional stresses on the substrate, which will result in erroneous contact angle value, which has often been the case so far ${ }^{4,5,11}$.

The second challenge for accurate quantification of the contact angle is to distinctly identify the demarcation contour between the oil drop base contact area and the fish scales, particularly more so in case of scales with mucus layer. As observed in case of existing literature ${ }^{3,5}$, the absence of clear demarcation results in possible inaccurate quantification of the contact angle, which is also shown in Fig. 4(c). Hence, proper illumination is required to accurately determine the drop base contact area. This requires modification of the existing DSA100 system, by bringing in an additional light source (Dolan-Jenner, USA.) with a diffuser (Edmund Optics, USA ), behind the glass couvette (Krüss, Germany), as shown in Fig. 4(b), so that accurate quantification of the contact angle can be obtained (refer Supplementary Video S2). Erroneous representation of contact angle due to improper illumination is presented in Fig. S1 (see Supplementary Information) and the repeatability in the measurement is depicted in Fig. S2 (see Supplementary Information).

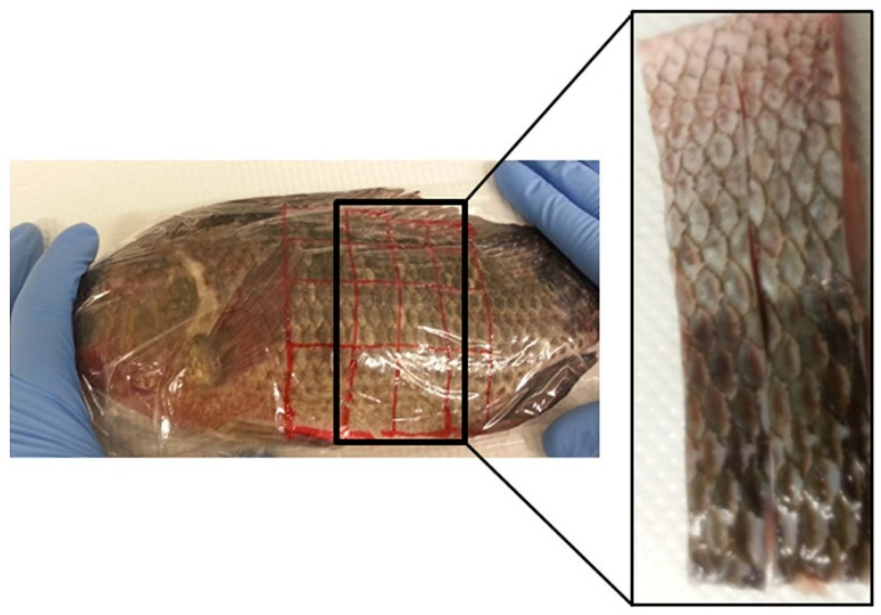

(a)
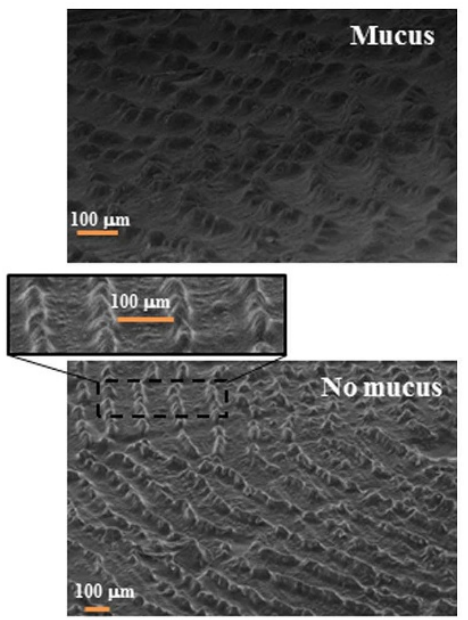

(b)

Figure 3 Tilapia fish used for quantification of wetting characteristics of its scales. The red marked parts of the skin was sectioned out for further analysis. (a) Sectioned out skin from the fish. (b) Scanning Electron Microscopy (SEM) image of fish scales with mucus and without mucus. 


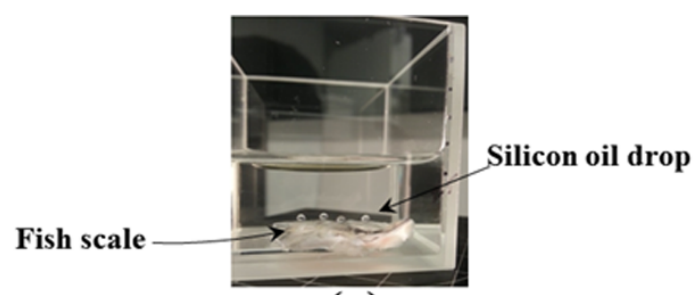

(a)

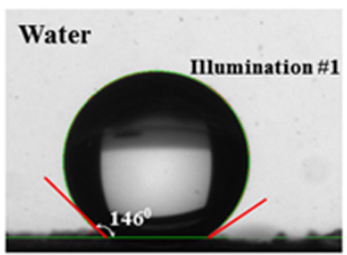

(c)

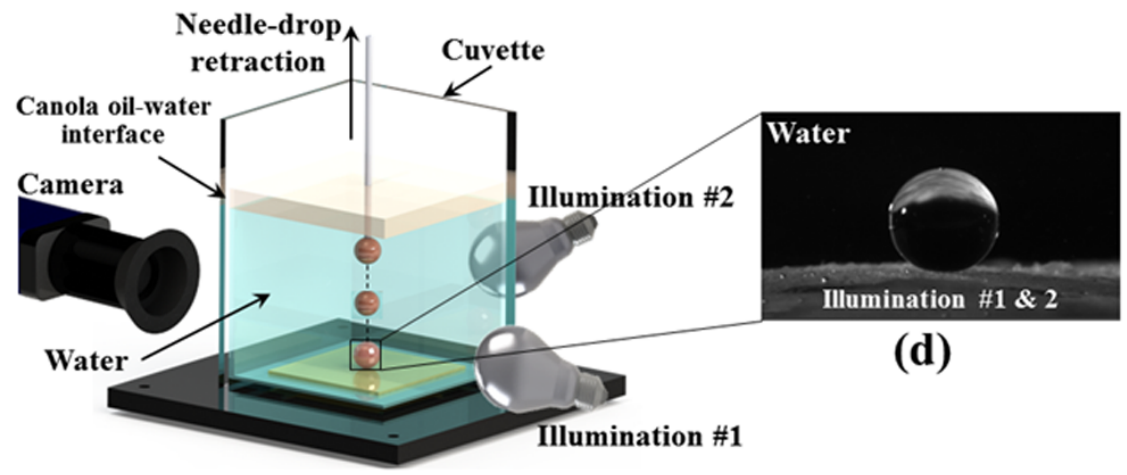

(b)

Figure $4 \mid$ Measurement of contact angle on fish scales. (a) Silicon oil drops, used as model fluid, resting on fish scales kept under water in a glass cuvette. (b) Technique used to achieve under-water needle-free drop deposition of silicon oil on soft deformable fish scale surface. (c) Commonly used procedure of contact angle measurement which inaccurately quantifies the static contact angle by using normal DSA 100 set-up due to unresolved drop base contact area. (d) Accurate quantification of contact angle using an additional illumination arrangement and modified experimental set-up. This figure was drawn by PRW.

Protocol for removal of mucus layer from fish scales. We have followed an established protocol ${ }^{16,17}$ for removing the mucus layer from the fish scales and we have further performed Fourier transform infrared spectroscopy (FTIR) to distinctly demarcate the presence and absence of mucus layer from the fish scales. All experimental protocols were approved by Risk Management Services, University of Alberta and methods were carried out in accordance with Biosafety Officer, Environment, Health and Safety Risk Management Services, University of Alberta. Dithiothreiotol (DDT) solution was prepared by dissolving $111 \mathrm{mg}$ of Ehtylenediamine tetracetic acid (EDTA) and $42 \mathrm{mg}$ of DTT in $300 \mathrm{ml}$ PBS. Sectioned out fish scales (see Fig. 3(a)) were kept for 30 mins. in DTT solution, after which the fish scales were rinsed with DI water to remove the residual DTT solution. Further, sufficient amount ( $100 \mathrm{ml}$ for $10 \mathrm{~cm} \times 10 \mathrm{~cm}$ sample) of dipco's modifies eagle medium (DMEM) was used to soak the fish scales for 30 mins. After removing the scales from DMEM solution, they were rinsed with DI water and kept completely soaked in the washing media $(90 \mathrm{ml} \mathrm{PBS}+5 \mathrm{ml}$ serum-research grade serum from Fisherbrand $+5 \mathrm{ml}$ P/S- Penicillin streptomycin, Gibco) for 30 mins. Finally, the fish scales were rinsed with another cycle of DI water and used for contact angle measurements.

ATR-FTIR spectroscopy. An Attenuated Total Reflection Fourier Transform Infrared (ATR-FTIR) spectroscope (Thermo Nicolet NEXUS 670 FTIR, USA) was used to obtain the FTIR data. Fish scale samples were kept on the ATR crystals in such a way that there is no air-gap between the fish scale and ATR crystal. At room temperature $\left(22^{\circ} \mathrm{C}\right), 128$ scans of $2 \mathrm{~cm}^{-1}$ were performed for samples with and without mucus. The data were collected and processed using Omnic software.

\section{Conclusion}

Here we have performed systematic static contact angle characterization of fish scales with and without mucus layer. The model fluid used is silicon oil and we have measured the contact angle of silicon oil on fish scales submerged in water using needle-free drop deposition technique coupled with proper illumination to decipher the drop contact area on the fish scale. This measurement technique allowed us to correctly quantify the contact angle for fish scales with mucus layer and we have observed an unprecedented contact angle of around $180^{\circ}$ for under-water fish scales. Also, through careful characterization of the mucus layer, we found that certain functional groups in mucus layer (hydroxyl (-OH), amine (-NH2) and carbonyl (CO)) are responsible for such extreme superoleophobicity of the fish scales. By removing the mucus layer, we found that the degree of superoleophobicity of the fish scales reduces significantly to a static contact angle of around $150^{\circ}$, which can then be attributed to the hierarchical micro/nano-structures on the fish scales. Hence, the present study conclusively proves that under-water superoleophobicity of fish scales is due to its inherent mucus layer formation, generated through its own defense mechanism (for e.g., foreign oil droplet coming in contact with its scales), rather than the common notion of the presence of hierarchical structures of the fish scales. This study further opens up new paradigm towards proper understanding of under-liquid wettability of such marine life, which is often been biomimicked through extensive micro/nanofabrication process to generate superoleophobic/superhydrophobic/omniphobic surfaces.

1. Shephard, K. L. Functions for mucus. Rev. Fish. Biol. Fisher 4, 401-429 (1994).

2. Bhushan, B. \& Jung, Y. C. Natural and biomimetic artificial surfaces for superhydrophobicity, self-cleaning, low adhesion, and drag reduction. Progr. Mater. Sci. 56, 1-108 (2011).

3. Bixler, G. D. \& Bhushan, B. Bioinspired rice leaf and butterfly wing surface structures combining shark skin and lotus effects. Soft Mat. 8, 11271-11284 (2012).

4. Koch, K., Bhushan, B. \& Barthlott, W. Diversity of structure, morphology and wetting of plant surfaces. Soft Mat. 4, 1943-1963 (2008).

5. Liu, M., Wang, S., Wei, Z., Song, Y. \& Jiang, L. Bioinspired Design of a Superoleophobic and Low Adhesive Water/Solid Interface. Adv. Mater. 21 665-669 (2009).

6. Nishimoto, S. \& Bhushan, B. Bioinspired self-cleaning surfaces with superhydrophobicity, superoleophobicity, and superhydrophilicity. RSC Adv. 3, 671-690 (2013).

7. Nosonovsky, M. \& Bhushan, B. Multiscale effects and capillary interactions in functional biomimetic surfaces for energy conversion and green engineering. Philos. Trans. R. Soc. London, Ser. A 367, 1511-1539 (2009).

8. Liu, M., Zheng, Y., Zhai, J. \& Jiang, L. Bioinspired Super-antiwetting Interfaces with Special Liquid-Solid Adhesion. Acc. Chem. Res. 43, 368-377 (2010).

9. Wainwright, S., Vosburgh, F. \& Herbrank, J. Shark Skin - Function in Locomotion. Science 202, 747-749 (1978).

10. Bixler, G. D. \& Bhushan, B. Bioinspired rice leaf and butterfly wing surface structures combining shark skin and lotus effects (vol 8, pg 11271, 2012). Soft Mat. 8, 12132-12144 (2012). 
11. Cai, Y. et al. Filefish-inspired surface design for anisotropic underwater oleophobicity. Adv. Funct. Mater. 24, 809-816 (2014).

12. Bixler, G. D. \& Bhushan, B. Biofouling: lessons from nature. Philos. Trans. R. Soc. London, Ser. A 370, 2381-2417 (2012).

13. Gennes, P.-G., de, Brochard-Wyart, F. \& Quéré, D. Capillarity and Wetting Phenomena: Drops, Bubbles, Pearls, Waves (Springer, France, 2002).

14. Smith, B. C. Fundamentals of Fourier Transform Infrared Spectroscopy (CRC Press, USA, 1996).

15. Waghmare, P. R., Das, S. \& Mitra, S. K. Drop deposition on under-liquid low energy surfaces. Soft Mat. 9, 7437-7447 (2013).

16. Leon, F. \& Roy, G. Isolation of human small bowel intraepithelial lymphocytes by Annexin V-coated magnetic beads. Lab. Invest. 84, 804-809 (2004).

17. Roche, J. K. Isolation of a purified epithelial cell population from human colon. Methods Mol. Biol. 50, 15-20 (2001).

\section{Acknowledgments}

The financial assistance from NSERC Discovery Grant program is acknowledged here. The authors would like to acknowledge the inputs from Dr. Selvaraj Naicker, Senior Research Associate in MNT Lab, University of Alberta, for discussions related to mucus layer in fish scales. The authors also like to acknowledge the contribution of Dr. Siddhartha Das, who is now an Assistant Professor in Mechanical Engineering, University of Maryland, towards writing the "Introduction" section of the manuscript and other valuable discussions that the authors had during the preparation of the manuscript.

\section{Author contributions}

S.K.M. conceived the problem and supervised the project. P.R.W. designed the entire experimental set up and performed all the experiments related to contact angle measurements and the FTIR analysis. NSKG provided the SEM images and cleaning protocol of fish samples. S.K.M. and P.R.W. wrote the paper.

\section{Additional information}

Supplementary information accompanies this paper at http://www.nature.com/ scientificreports

Competing financial interests: The authors declare no competing financial interests.

How to cite this article: Waghmare, P.R., Gunda, N.S.K. \& Mitra, S.K. Under-water superoleophobicity of fish scales. Sci. Rep. 4, 7454; DOI:10.1038/srep07454 (2014).

(c) (1) $(-)$ This work is licensed under a Creative Commons Attribution-NonCommercialNoDerivs 4.0 International License. The images or other third party material in this article are included in the article's Creative Commons license, unless indicated otherwise in the credit line; if the material is not included under the Creative Commons license, users will need to obtain permission from the license holder in order to reproduce the material. To view a copy of this license, visit http:// creativecommons.org/licenses/by-nc-nd/4.0/ 